\title{
Size levels for arcs
}

\author{
by \\ Sam B. Nadler, J r. (Morgantown, W.Va.) \\ and Thelma West (Lafayette, La.)
}

\begin{abstract}
We determine the size levels for any function on the hyperspace of an arc as follows. Assume $Z$ is a continuum and consider the following three conditions: 1) $Z$ is a planar AR; 2) cut points of $Z$ have component number two; 3) any true cyclic element of $Z$ contains at most two cut points of $Z$. Then any size level for an arc satisfies 1 ) -3 ) and conversely, if $Z$ satisfies 1 ) -3 ), then $Z$ is a diameter level for some arc.
\end{abstract}

1. Introduction. Let $X$ be a continuum and let $C(X)$ denote the hyperspace of subcontinua of $X\left[\mathrm{~N}_{1}\right.$, p. 1]. A Whitney map for $C(X)$ is a continuous function $\mu: C(X) \rightarrow[0,+\infty)$ such that $\mu(\{x\})=0$ and if $A \subset B$ and $A \neq B$ then $\mu(A)<\mu(B)$. Much work has been done in the study of Whitney levels (point inverses) of Whitney maps. More generally, let us call a continuous function $\sigma: C(X) \rightarrow[0,+\infty)$ a size map provided that $\sigma(\{x\})=0$ and, if $A \subset B, \sigma(A) \leq \sigma(B)$. For example, the diameter map is a size map which is not in general a Whitney map. Point inverses of size maps are called size levels. Whitney levels are continua [EN, p. 1032] and, by the same proof as in [EN, p. 1032], size levels are continua. Whitney levels for arcs are arcs (or degenerate). In this paper we shall determine the size levels for any size function on the hyperspace of an arc as follows:

Theorem. Assume $Z$ is a continuum and consider the following three conditions:

1.1. $Z$ is a planar $A R$.

1.2. Cut points of $Z$ have component number two.

1.3. Any true cyclic element of $Z$ contains at most two cut points of $Z$.

Then any size level for an arc satisfies 1.1-1.3 and conversely, if $Z$ satisfies 1.1-1.3, then $Z$ is a diameter level for some arc.

1991 Mathematics Subject Classification: Primary 54F20; Secondary 54F30.

Key words and phrases: hyperspace, cyclic elements, absolute retract. 
We prove this theorem in Sections 3-6. In Section 7, we give an example of a Peano continuum which has a diameter level which is not locally connected. This is in contrast to the case of Whitney levels which, for Peano continua, must again be Peano continua [ $\mathrm{N}_{2}$, Th. 3].

2. Some general terminology and notation. Most of our terminology and notation is standard or will be explained later. We note here the following general definitions.

A Peano continuum is a locally connected continuum (continuum meaning nonempty, compact, connected metric space). The notion of a cyclic element may be found in $[\mathrm{K}]$ or $[\mathrm{W}]$.

If $S$ is a connected space and $p \in S$, then the component number of $p$ (in $S$ ) is the cardinality of the set of all components of $S-\{p\}$. We say $p$ is a cut point of $S$ provided that $S-\{p\}$ is not connected, i.e., the component number of $p$ in $S$ is $\geq 2$.

The symbol $\bar{A}$ denotes the closure of $A$. If $H \subset X$, then $\operatorname{Bd}(H)$ denotes the (topological) boundary of $H$ in $X$, i.e., $\operatorname{Bd}(H)=\bar{H} \cap \overline{X-H}$.

An $\operatorname{arc} A$ in $X$ with end points $p$ and $q$ is called a free $\operatorname{arc}($ in $X)$ provided that $A-\{p, q\}$ is open in $X$.

We shall let $|A|$ denote the cardinality of a set $A$.

3. Proof of necessity of 1.1-1.3. Let $I$ be the unit interval with any metric $d$. Then $C(I)$ is a 2-cell (see $\left.\left[\mathrm{N}_{1}\right]\right)$. The geometric representation we use for $C(I)$ is the set $\Gamma$ given by $\left(I^{2}=I \times I\right)$

$$
\Gamma=\left\{(x, y) \in I^{2}: y \geq x\right\}
$$

where $(x, y)$ represents the subarc $[x, y]$ of $I$ when $x \neq y$ and $(x, x)$ represents the set $\{x\}$. A point in a size level $L_{t}$ will be thought of interchangeably as a subcontinuum of $I$ or as a point in $\Gamma$.

We define a map $\pi: C(I) \rightarrow D$, where $D$ is the diagonal of $I^{2}$, by

$$
\pi(x, y)=((x+y) / 2,(x+y) / 2) .
$$

Also, for each $z \in I$, let

$$
F_{z}=\{(x, y) \in \Gamma:(x+y) / 2=z\}=\pi^{-1}(z, z) .
$$

Let $y_{t}=\min \left\{y \in I:(0, y) \in L_{t}\right\}$ and $x_{t}=\max \left\{x \in I:(x, 1) \in L_{t}\right\}$. Let $\pi_{t}=\pi \mid L_{t}$.

3.1. Box Lemma. Let $z \in[0,1]$. If $p, q \in F_{z} \cap L_{t}$ where $p=(x, y)$ and $q=\left(x^{\prime}, y^{\prime}\right)$, then $\left[x, x^{\prime}\right] \times\left[y^{\prime}, y\right] \subset L_{t}$.

Proof. Let $r \in\left[x, x^{\prime}\right]$ and $s \in\left[y^{\prime}, y\right]$. Then we see immediately that

$$
\left[x^{\prime}, y^{\prime}\right] \subset[r, s] \subset[x, y] .
$$

Thus, since $p, q \in L_{t}$, clearly $[r, s] \in L_{t}$. This proves 3.1 . 
3.2. Lemma. The map $\pi_{t}: L_{t} \rightarrow D$ is monotone (but not necessarily onto $D)$. In fact, for any $(z, z) \in \pi_{t}\left(L_{t}\right), \pi_{t}^{-1}(z, z)$ is a one-point set or an arc.

Proof. By 3.1, $\pi_{t}^{-1}(z, z)$ is a connected subset of $F_{z} \cap L_{t}$.

3.3. Lemma. For each $(x, y) \in L_{t}, y_{t} \leq y$ and $x \leq x_{t}$.

Proof. If $y \leq y_{t}$, then $[x, y] \subset[0, y] \subset\left[0, y_{t}\right]$. Thus, since $[x, y]$, $\left[0, y_{t}\right] \in L_{t},[0, y] \in L_{t}$. Hence, $y=y_{t}$. If $x_{t} \leq x$, then $[x, y] \subset[x, 1] \subset\left[x_{t}, 1\right]$. Thus, since $[x, y],\left[x_{t}, 1\right] \in L_{t},[x, 1] \in L_{t}$. Hence, $x_{t}=x$.

3.4. Lemma. Each of the points $\left(0, y_{t}\right)$ and $\left(x_{t}, 1\right)$ are noncut points of $L_{t}$.

Proof. Note that $\pi_{t}\left(L_{t}\right)$ is the arc in $D$ with end points $\pi_{t}\left(0, y_{t}\right)$ and $\pi_{t}\left(x_{t}, 1\right)$. Also note that

$$
\pi_{t}^{-1}\left(\pi_{t}\left(0, y_{t}\right)\right)=\left\{\left(0, y_{t}\right)\right\} \quad \text { and } \quad \pi_{t}^{-1}\left(x_{t}, 1\right)=\left\{\left(x_{t}, 1\right)\right\} \text {. }
$$

Hence, the lemma follows easily from 3.2 and from 2.2 of [W, p. 138].

3.5. Lemma. A point $p=(x, y)$ is a cut point of $L_{t}$ if and only if $p \neq$ $\left(0, y_{t}\right), p \neq\left(x_{t}, 1\right)$, and $\pi_{t}^{-1}\left(\pi_{t}(p)\right)=\{p\}$.

Proof. Assume $p \neq\left(0, y_{t}\right), p \neq\left(x_{t}, 1\right)$, and $\pi_{t}^{-1}\left(\pi_{t}(p)\right)=\{p\}$. Then the line $Q$ of slope -1 in $\Gamma$ through $\pi(p)$ separates $\left(0, y_{t}\right)$ and $\left(x_{t}, 1\right)$ in $\Gamma$ by 3.3. Thus, since

$$
Q \cap L_{t}=\pi_{t}^{-1}\left(\pi_{t}(p)\right)=\{p\},
$$

we see that $p$ is a cut point of $L_{t}$. To prove the other half of the lemma, assume $p$ is a cut point of $L_{t}$. By 3.4, $p \neq\left(0, y_{t}\right)$ and $p \neq\left(x_{t}, 1\right)$. Let $\pi_{t}\left(L_{t}\right)=A$. Recall that $A$ is an arc with end points $\pi\left(0, y_{t}\right)$ and $\pi\left(x_{t}, 1\right)$. Thus, since $p \neq\left(0, y_{t}\right)$ and $p \neq\left(x_{t}, 1\right), A-\left\{\pi_{t}(p)\right\}$ has exactly two components $A_{1}$ and $A_{2}$. By 3.2 (and by 2.2 of [W, p. 138]), $\pi_{t}^{-1}\left(A_{1}\right)$ and $\pi_{t}^{-1}\left(A_{2}\right)$ are connected. By the Box Lemma 3.1, any point in $\pi_{t}^{-1}\left(\pi_{t}(p)\right)-\{p\}$ would be a limit point of both $\pi_{t}^{-1}\left(A_{1}\right)$ and $\pi_{t}^{-1}\left(A_{2}\right)$. Hence, if

$$
\pi_{t}^{-1}\left(\pi_{t}(p)\right)-\{p\} \neq \emptyset
$$

then $L_{t}-\{p\}$ would be connected, in contradiction to our assumption that $p$ is a cut point of $L_{t}$. Therefore, $\pi_{t}^{-1}\left(\pi_{t}(p)\right)=\{p\}$. This completes the proof of 3.5 .

3.6. Lemma. Each $L_{t}$ is a retract of $C(I)$.

Proof. Let $A=\{(x, y) \in \Gamma$ : the straight line $Q(x, y)$ in $\Gamma$ through $(x, y)$ of slope -1 intersects $\left.L_{t}\right\}$. For any $(x, y) \in A$, let $h(x, y)$ denote the highest point of $Q(x, y) \cap L_{t}$ (i.e., the point of $Q(x, y) \cap L_{t}$ with largest $y$-coordinate), and let $l(x, y)$ denote the lowest point of $Q(x, y) \cap L_{t}$ (i.e., the point of $Q(x, y) \cap L_{t}$ with smallest $y$-coordinate). It is easy using the Box Lemma to see that if $\left\{\left(x_{i}, y_{i}\right)\right\}_{i=1}^{\infty}$ is a sequence in $A$ converging to $(x, y) \in A$, then 
$\left\{h\left(x_{i}, y_{i}\right)\right\}_{i=1}^{\infty}$ converges to $h(x, y)$ and $\left\{l\left(x_{i}, y_{i}\right)\right\}_{i=1}^{\infty}$ converges to $l(x, y)$. Thus, $h$ and $l$ are continuous on $A$. We define a retraction $r$ from $\Gamma$ onto $L_{t}$ as follows. Fix $(x, y) \in \Gamma$, and let $Q$ denote the straight line in $\Gamma$ through $(x, y)$ of slope -1 . If $(x, y) \in L_{t}$, let $r(x, y)=(x, y)$. If $(x, y) \notin L_{t}$ and $Q \cap L_{t} \neq \emptyset$, then we see by 3.2 that (1) $y>$ second coordinate of $h(x, y)$ or (2) $y<$ second coordinate of $l(x, y)$; if (1) holds, let $r(x, y)=h(x, y)$, and if (2) holds, let $r(x, y)=l(x, y)$. Finally, if $Q \cap L_{t}=\emptyset$, let $r(x, y)=\left(0, y_{t}\right)$ if $y \leq-x+y_{t}$ and let $r(x, y)=\left(x_{t}, 1\right)$ if $y \geq-x+x_{t}+1$. Thus, we have defined a function $r$ on all of $C(I)$ to $L_{t}$. The continuity of $r$ follows from the continuity of $h$ and $l$ on $A$. Therefore, $r$ is our desired retraction from $\Gamma$ onto $L_{t}$.

\subsection{Lemma. Cut points of $L_{t}$ have component number two.}

Proof. This lemma follows immediately from 3.2 and 3.5 by using 2.2 of [W, p. 138].

3.8. Lemma. Assume $H$ is a true cyclic element of $L_{t}$, and assume $p$ and $q$ are cut points of $L_{t}$ such that $p, q \in H$ and $p \neq q$. Let $J$ be the arc in $D$ with end points $\pi_{t}(p)$ and $\pi_{t}(q)$ (note $\pi_{t}(p) \neq \pi_{t}(q)$ by 3.5). Then $H=\pi_{t}^{-1}(J)$.

Pro of. Since $H$ is connected and $p, q \in H$, clearly $\pi_{t}(H) \supset J$. Suppose there exists $r \in H$ such that $\pi_{t}(r) \notin J$. Then one of $\pi_{t}(p)$ or $\pi_{t}(q)$, say $\pi_{t}(q)$, separates the other point from $\pi_{t}(r)$ in $D$. Hence, by 3.5, $q$ separates $p$ from $r$ in $L_{t}$. Therefore, $q$ is a cut point of $H$, in contradiction to $H$ being a cyclic element. Hence, $\pi_{t}(H) \subset J$. This completes the proof of 3.8.

3.9. Lemma. Each true cyclic element of $L_{t}$ contains at most two cut points of $L_{t}$.

Proof. The lemma follows immediately from 3.8 .

By 3.6, 3.7 and 3.9, we have proved the necessity of 1.1-1.3.

\section{Preliminary lemmas about cyclic elements}

4.1. Lemma. Assume $Z$ satisfies 1.1 and 1.2. If $v$ is the vertex of a simple triod $K$ in $Z$, then $v$ is a point of a true cyclic element of $Z$.

Proof. Using 1.2 in the case when $v$ is a cut point of $Z$, we see that there is an arc in $Z-\{v\}$ irreducible between points of two different legs of $K$. Hence, using 1.1, we see that $v$ is a point of a two-cell and, therefore, $v$ belongs to a true cyclic element of $Z$.

4.2. Lemma. Assume $Z$ satisfies 1.1 and 1.2. If $p$ and $q$ are distinct cut points of $Z$, then either (1) $p$ and $q$ are end points of a free arc, (2) $p$ and $q$ 
are in the same true cyclic element of $Z$, or (3) there is a point $w$ of a true cyclic element of $Z$ such that $w$ separates $p$ and $q$ in $Z$.

Proof. Let $A$ be an arc in $Z$ from $p$ to $q$. Assume (1) is false. Then, by 4.1 , there is a true cyclic element $T$ of $Z$ such that

$$
T \cap(A-\{p, q\}) \neq \emptyset .
$$

Assume (2) is false. Then at least one of $p$ or $q$, say $q$, is not an element of $T$. Hence, there is a point $w$ such that

$$
w \in \operatorname{Bd}(T) \cap(A-\{p, q\}) .
$$

Now, suppose (3) is false, i.e., suppose $p$ and $q$ are in the same component of $Z-\{w\}$. Then there is an $\operatorname{arc} B$ from $p$ to $q$ such that $w \notin B$. It is easy to see that there is an arc $E \subset A \cup B$ such $E \cap T$ is not connected. This contradicts 3.4 and 3.5 of [W, p. 69].

4.3. Lemma. Assume $Z$ satisfies 1.1 and 1.2 and that $p$ and $q$ are distinct cut points of $Z$ such that no point of any true cyclic element of $Z$ separates $p$ and $q$ in $Z$. If $A$ is an arc in $Z$ from $p$ to $q$ then either $A$ is contained in a true cyclic element of $Z$ or $A$ is a free arc in $Z$.

Proof. Assume $A$ is not a free arc in $Z$. Then (1) of 4.2 is false since if there were a free arc $F$ in $Z$ from $p$ to $q, A \cup F$ would be a simple closed curve containing a free arc and, therefore, $A \cup F$ would be a retract of $Z$ which would contradict 1.1. Thus, (2) of 4.2 must hold. Hence, by 3.4 and 3.5 of [W, p. 69], $A$ is contained in a true cyclic element of $Z$.

4.4. Lemma. Assume $Z$ is a Peano continuum satisfying 1.3. If $T$ is a true cyclic element of $Z$, then each point of $\operatorname{Bd}(T)$ is a cut point of $Z$ (thus, $|\operatorname{Bd}(T)| \leq 2$ ).

Proof. Suppose $p \in \operatorname{Bd}(T)$ such that $p$ is a noncut point of $Z$. Then, by the local arcwise connectedness of $Z$ and 1.3, there is an $\operatorname{arc} A$ from a point $x \in Z-T$ to a point $q \in T$ (near $p$ ) so that $A \cap T=\{q\}$ and $q$ is a noncut point of $Z$. Now, there is an arc $B$ in $Z-\{q\}$ from $x$ to a point $r$ in $T$. Clearly, $A \cup B$ contains an arc $E$ such that $|E \cap T|=2$. This contradicts 3.4 and 3.5 of [W, p. 69]. This proves 4.4 .

4.5. Lemma. Assume $Z$ is nondegenerate and satisfies 1.1-1.3. Then there exist distinct points $p_{l}$ and $p_{r}$ of $Z$ such that every cut point separates $p_{l}$ and $p_{r}$.

Proof. Assume there is a cut point of $Z$. Then, by 8.2 of [W, p. 77], $Z$ has at least two nodes $N_{l}$ and $N_{r}$. Let $p_{l} \in N_{l}$ and $p_{r} \in N_{r}$ such that $p_{l}$ and $p_{r}$ are noncut points of $Z$. Suppose there is a cut point $c$ of $Z$ such that $c$ does not separate $p_{l}$ and $p_{r}$ in $Z$. Note that $c \notin N_{l} \cup N_{r}$ since $c$ is a cut point of $Z$ and cannot be the boundary point of $N_{l}$ or $N_{r}$. Then there 
is an $\operatorname{arc} A$ in $Z-\{c\}$ from $p_{l}$ to $p_{r}$. Since $p_{l}$ and $p_{r}$ are either end points of $Z$ or non-boundary points of nodal sets, $p_{l} \notin N_{r}$ and $p_{r} \notin N_{l}$. Thus,

$$
\operatorname{Bd}\left(N_{l}\right) \cap A \neq \emptyset \neq \operatorname{Bd}\left(N_{r}\right) \cap A .
$$

Hence

$$
\operatorname{Bd}\left(N_{l}\right) \cap A=\operatorname{Bd}\left(N_{l}\right) \text { and } \operatorname{Bd}\left(N_{r}\right) \cap A=\operatorname{Bd}\left(N_{r}\right) .
$$

Since $c \notin N_{l} \cup N_{r}$, there is an arc $B$ from $c$ to a point $w$ of $A$ such that $A \cap B=\{w\}$. By (2),

$$
(B-\{w\}) \cap\left(N_{l} \cup N_{r}\right)=\emptyset .
$$

If $w \in \operatorname{Bd}\left(N_{l}\right) \cup \operatorname{Bd}\left(N_{r}\right)$, then at least two of $N_{l}-\operatorname{Bd}\left(N_{l}\right), N_{r}-\operatorname{Bd}\left(N_{r}\right)$, and $c$ are, by 1.2 , in the same component of $Z-\{w\}$ and, therefore, it follows that at least one of $N_{l}$ or $N_{r}$ has a boundary point not in $A$, which is a contradiction to (2). Assume

$$
w \notin \operatorname{Bd}\left(N_{l}\right) \cup \operatorname{Bd}\left(N_{r}\right) .
$$

Then, by 4.1, $w$ is a point of some true cyclic element $H$ of $Z$. Recalling that $c$ is a cut point of $Z$, it follows easily that either $w$ does not satisfy 1.2 or $H$ does not satisfy 1.3 . This proves 4.5 .

For each $Z$ satisfying 1.1 through 1.3 , we shall construct an $\operatorname{arc} A$ in the plane with the max metric $d$,

$$
d\left(\left(x_{1}, y_{1}\right),\left(x_{2}, y_{2}\right)\right)=\max \left\{\left|x_{2}-x_{1}\right|,\left|y_{2}-y_{1}\right|\right\},
$$

such that $Z$ is a diameter level for $A$. In the next two sections we shall use the set $C$ defined as follows:

$$
\begin{aligned}
C=\{c \in Z: & c \text { is a cut point of } Z \text { and either } \\
& c \text { is a point of a true cyclic element of } Z \text { or } \\
& c \text { is an end point of a maximal free } \operatorname{arc} \text { of } Z\} .
\end{aligned}
$$

We shall first consider the case where the number of true cyclic elements is finite.

5. Finite case. For convenience we first note the following special case:

Case 1: $Z$ is a point, an arc or a 2-cell. Let $I=\{(x, y): 0 \leq x \leq 1$, $y=0\}$. Then $L_{0}(I)$ is an arc and $L_{1}(I)$ is a point. If $Z$ is a 2-cell, then let

$$
A=([1 / 2,1] \times\{0\}) \cup(\{1\} \times[0,1]) \cup([1,3 / 2] \times\{1\}) .
$$

Then $L_{1}(A)$ is homeomorphic to $Z$.

Next, we consider the following case:

Case 2: $Z$ has at least one and at most a finite number of true cyclic elements and $Z$ contains a cut point which is a point of a true cyclic element of $Z$. We see that Case 2 covers all the finite cases that were not considered 
in Case 1 as follows. Suppose that $Z$ does not satisfy the conditions of Case 2. Then either $Z$ contains no true cyclic element or $Z$ contains no cut point which is a point of a true cyclic element of $Z$. If $Z$ contains no true cyclic element, then, by [W, p. 89] and 1.1, $Z$ must be a dendrite or a point. Assume $Z$ is nondegenerate. Then, by 1.2 , [W, Th. 6.1, p. 54] and [W, Th. 1.1(ii), p. 88], $Z$ has exactly two noncut points. Hence, $Z$ is an arc. If $Z$ contains a true cyclic element $T$ such that $Z-T$ is nonempty, then, by $[\mathrm{W}, 2.1$, p. 66], $T$ contains a cut point of $Z$. Consequently, by our assumption, $Z=T$ and thus by 1.1 and [K, p. 534], $Z$ is a 2-cell.

Assume that $Z$ is a continuum satisfying 1.1-1.3 and the conditions of this case. Then the set $C$ is finite and we let $n$ be the cardinality of $C$. We can label the points of $C$ by $c_{1}, \ldots, c_{n}$, using 4.5 and the following scheme. Let $c_{1}$ be the element of $C$ such that $C \cap N_{l}=\emptyset$ where $N_{l}$ is the component of $Z-\left\{c_{1}\right\}$ containing $p_{l}$. In general for $i=2, \ldots, n$, let $c_{i}$ be the point of $C$ such that $C \cap N_{i}=\left\{c_{1}, \ldots, c_{i-1}\right\}$ where $N_{i}$ is the component of $Z-\left\{c_{i}\right\}$ containing $p_{l}$. We shall represent $Z$ by an $(n+1)$-tuple of points $\left(x_{1}, \ldots, x_{n+1}\right)$ as follows. Let $p_{l}=c_{0}$ and $p_{r}=c_{n+1}$. For a given $i=1, \ldots, n-1$, we let $x_{i}=2$ if $c_{i-1}$ and $c_{i}$ are points of the same true cyclic element of $Z$ and we let $x_{i}=1$ if $c_{i-1}$ and $c_{i}$ are the end points of a maximal free arc in $Z$. Note that by 4.2 , this defines $x_{i}$ for each $i$.

Let $t$ be the number of true cyclic elements in $Z$, and let $f$ be the number of maximal free $\operatorname{arcs}$ in $Z$; thus $t$ is the number of 2 's and $f$ is the number of 1 's in $\left(x_{1}, \ldots, x_{n+1}\right)$. We define numbers $v$ and $h$ as follows. First, let $k$ be the greatest integer less than or equal to $t / 2$. Then let $v=k+f$, let $h=k+f$ if $t=2 k$, and let $h=k+f+1$ if $t=2 k+1$.

Let $Y=([0,1] \times[0,1]) \cup([1,2] \times[1,2]) \cup([2,3] \times[2,3])$. We shall construct an $\operatorname{arc} A$ in $Y$ such $L_{2}(A)$ is homeomorphic to $Z$. First, we construct $n+1$ arcs such that the union of these arcs is an arc in $[0,1] \times[0,1]$ with end points $(0,0)$ and $(1,1)$. Let $e_{0}^{1}=(0,0)$. If $x_{1}=1$, let $e_{1}^{1}=(1 / h, 1 / v)$. If $x_{1}=2$, let $e_{1}^{1}=(1 / h, 0)$. Let $A^{1}=e_{0}^{1} e_{1}^{1}$, the convex arc from $e_{0}^{1}$ to $e_{1}^{1}$. We define $A^{k}$ for $k=2, \ldots, n+1$ by induction. Assume we have defined $e_{0}^{j} e_{1}^{j}$. We let $e_{0}^{j+1}=e_{1}^{j}$. If $x_{j+1}=1$, let $e_{1}^{j+1}=e_{0}^{j+1}+(1 / h, 1 / v)$. If $x_{j+1}=2$ and the number of coordinates which are 2 in $\left(x_{1}, \ldots, x_{j}\right)$ is even, let $e_{1}^{j+1}=e_{0}^{j+1}+(1 / h, 0)$. If $x_{j+1}=2$ and the number of coordinates which are 2 in $\left(x_{1}, \ldots, x_{j}\right)$ is odd, let $e_{1}^{j+1}=e_{0}^{j+1}+(0,1 / v)$. Now, let $A^{j+1}=e_{0}^{j+1} e_{1}^{j+1}$.

It is clear that $\bigcup_{i=1}^{n+1} A^{i}$ is an arc. We show that $\bigcup_{i=1}^{n+1} A^{i} \subset[0,1] \times[0,1]$ with end points $(0,0)$ and $(1,1)$ as follows. If $t=2 k$ then

$$
\begin{aligned}
e_{1}^{n+1} & =(0,0)+f\left(\frac{1}{h}, \frac{1}{v}\right)+k\left(\frac{1}{h}, 0\right)+k\left(0, \frac{1}{v}\right) \\
& =\left(\frac{f+k}{h}, \frac{f+k}{v}\right)=(1,1) .
\end{aligned}
$$


If $t=2 k+1$ then

$$
\begin{aligned}
e_{1}^{n+1} & =(0,0)+f\left(\frac{1}{h}, \frac{1}{v}\right)+(k+1)\left(\frac{1}{h}, 0\right)+k\left(0, \frac{1}{v}\right) \\
& =\left(\frac{f+k+1}{h}, \frac{f+k}{v}\right)=(1,1) .
\end{aligned}
$$

In both cases, $e_{1}^{n}=(1,1)$. Therefore, $(0,0)$ and $(1,1)$ are the end points of $B=\bigcup_{i=1}^{n+1} A^{i}$ and hence $B \subset[0,1] \times[0,1]$. Now, let

$$
A=B \cup\{(x, x): x \in[1,2]\} \cup\{(x+2, y+2):(x, y) \in B\} .
$$

By inspection we can see that $L_{2}(A)$ is homeomorphic to $Z$ (recall that we are using the max metric $d$ ).

For use later on, note the following two facts.

5.1. In the construction above, we could have chosen the first horizontal arc to have been vertical (and make appropriate changes for the other arcs).

5.2. We shall use the following terminology. An admissible staircase is an $\operatorname{arc} B$ constructed as above (consisting of horizontal, vertical and diagonal segments) but perhaps in a rectangle or in a square other than $[0,1] \times[0,1]$.

6. Infinite case. In this case the set $C$ associated with $X$ is countably infinite. We construct an $\operatorname{arc} B$ in $I \times I$ and an $\operatorname{arc} A$ by

$$
A=B \cup\{(x, x): x \in[1,2]\} \cup\{(x+1, y+1):(x, y) \in B\}
$$

and show that $L_{2}(A)$ is homeomorphic to $Z$.

Pick $c_{1} \in C$. Then $X-\left\{c_{1}\right\}=U_{0} / U_{\infty}$ where $p_{r} \in U_{0}$ and $p_{l} \in U_{\infty}$.

We begin the construction of $B$ in (a)-(g) below.

(a) $U_{0} \cap C$ is nonempty and finite. Then let $A_{1}$ be an admissible staircase in $[0,1 / 2] \times[0,1 / 2]$ with end points $(0,0)$ and $(1 / 2,1 / 2)$.

(b) $U_{\infty} \cap C$ is nonempty and finite. Let $A_{1}$ be an admissible staircase in $[1 / 2,1] \times[1 / 2,1]$ with end points $(1 / 2,1 / 2)$ and $(1,1)$.

(c) $U_{0} \cap C=\emptyset$ and $U_{0} \cup\left\{c_{1}\right\}$ is a free arc. Let $A_{1}=(0,0)(1 / 2,1 / 2)$.

(d) $U_{\infty} \cap C=\emptyset$ and $U_{\infty} \cup\left\{c_{1}\right\}$ is a free arc. Let $A_{1}=(1 / 2,1 / 2)(1,1)$.

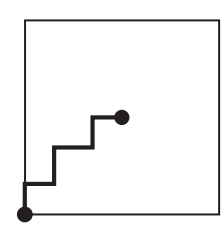

a

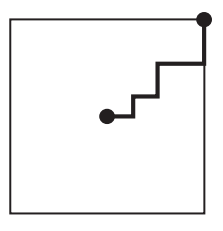

$\mathrm{b}$

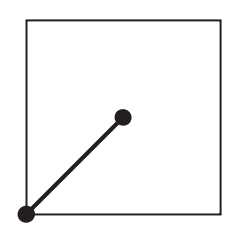

$\mathrm{C}$

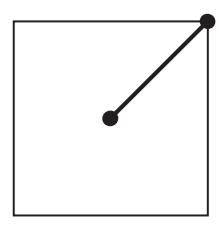

d

(e) $U_{0} \cap C=\emptyset$ and $U_{0} \cup\left\{c_{1}\right\}$ is a true cyclic element of $Z$. Then let $A_{1}=\{0\} \times[0,1 / 2]$. Now pick $c_{2} \in C-\left\{c_{1}\right\}$. Let $X-\left\{c_{2}\right\}=V_{0} / V_{\infty}$. We consider the following subcases. 
$\left(\mathrm{e}_{1}\right) V_{\infty} \cap C=\emptyset$ and $V_{\infty} \cup\left\{c_{2}\right\}$ is a free arc. Let $A_{2}=(1 / 4,3 / 4)(1,1)$.

$\left(\mathrm{e}_{2}\right) V_{\infty} \cap C=\emptyset$ and $V_{\infty} \cup\left\{c_{2}\right\}$ is a true cyclic element of $Z$. Then let $A_{2}=[1 / 2,1] \times\{1\}$.

(e $\left.\mathrm{e}_{3}\right) V_{\infty} \cap C$ is nonempty and finite. Then let $A_{2}$ be an admissible staircase in $[1 / 4,1] \times[3 / 4,1]$ with end points $(1 / 4,3 / 4)$ and $(1,1)$.
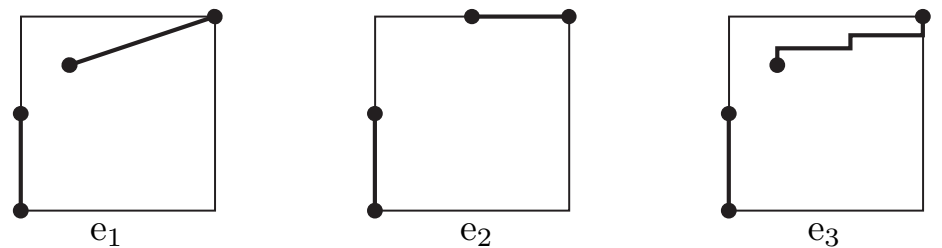

$\left(\mathrm{e}_{4}\right) V_{0} \cap C=\left\{c_{1}\right\}$ and $c_{1}$ and $c_{2}$ are end points of the same maximal free arc. Let $A_{2}=(0,1 / 2)(3 / 4,3 / 4)$.

(e $\left.\mathrm{e}_{5}\right) V_{0} \cap C=\left\{c_{1}\right\}$ and $c_{1}$ and $c_{2}$ are end points of the same true cyclic element of $Z$. Let $A_{2}=(0,1 / 2)(1 / 2,1 / 2)$.

( $\left.\mathrm{e}_{6}\right) V_{0} \cap C-\left\{c_{1}\right\}$ is nonempty and finite. Then let $A_{2}$ be an admissible staircase in $[0,3 / 4] \times[1 / 2,3 / 4]$ with end points $(0,1 / 2)$ and $(3 / 4,3 / 4)$.
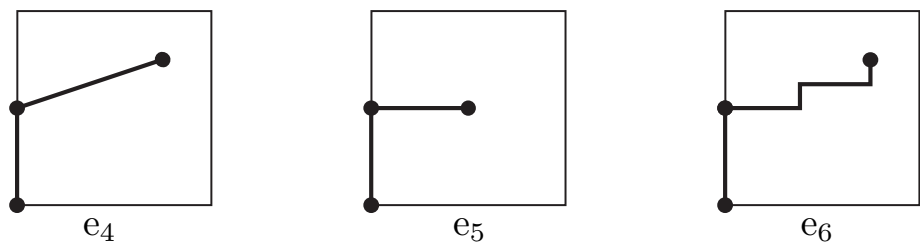

$\left(\mathrm{e}_{7}\right) V_{0} \cap C$ and $V_{\infty} \cap C$ are both infinite. Then we pick a point $c_{3} \in C$ such that either $\left(\mathrm{e}_{7 \mathrm{a}}\right) c_{2}$ and $c_{3}$ are the end points of a maximal free arc, or $\left(\mathrm{e}_{7 \mathrm{~b}}\right) c_{1}$ and $c_{2}$ are points of the same true cyclic element of $Z$. By the definition of $C$ we can pick a point $c_{3}$ satisfying either $\left(\mathrm{e}_{7 \mathrm{a}}\right)$ or $\left(\mathrm{e}_{7 \mathrm{~b}}\right)$.

If $\left(\mathrm{e}_{7 \mathrm{a}}\right)$ holds, then let $A_{2}=(1 / 8,5 / 8)(7 / 8,7 / 8)$. If ( $\left.\mathrm{e}_{7 \mathrm{~b}}\right)$ holds, then let $A_{2}=[1 / 4,3 / 4] \times\{3 / 4\}$.
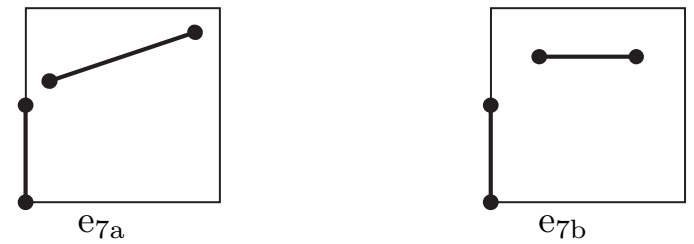

(f) $U_{\infty} \cap C=\emptyset$ and $U_{\infty} \cup\left\{c_{1}\right\}$ is a true cyclic element of $Z$. This case is similar to $(\mathrm{e})$.

By (a)-(f) only one general case remains to be considered for the first step in this construction.

(g) $U_{0} \cap C$ and $U_{\infty} \cap C$ are infinite. Then pick $c_{2} \in C$ such that either 
$\left(\mathrm{g}_{\mathrm{a}}\right) c_{1}$ and $c_{2}$ are end points of a maximal free arc, or $\left(\mathrm{g}_{\mathrm{b}}\right) c_{1}$ and $c_{2}$ are points of the same true cyclic element of $Z$. By the definition of $C$ we can pick a point $c_{2}$ satisfying either $\left(\mathrm{g}_{\mathrm{a}}\right)$ or $\left(\mathrm{g}_{\mathrm{b}}\right)$. We shall assume without loss of generality, by reindexing $c_{1}$ and $c_{2}$, that $c_{1}$ separates $p_{l}$ and $c_{2}$ in $Z$.

If $\left(\mathrm{g}_{\mathrm{a}}\right)$ holds, then let $A_{1}=(1 / 4,1 / 4)(3 / 4,3 / 4)$. If $\left(\mathrm{g}_{\mathrm{b}}\right)$ holds, then let $A_{1}=\{1 / 2\} \times[1 / 4,3 / 4]$.
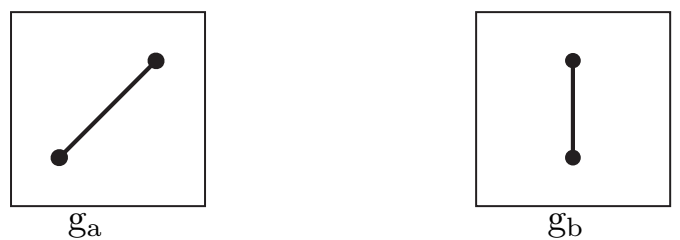

Then pick $c_{3} \in C$ such that $c_{3}$ separates $p_{l}$ and $c_{1}$ in $Z$. Let $X-\left\{c_{3}\right\}=$ $V_{0} / V_{\infty}$ where $p_{l} \in V_{0}$ and $p_{r} \in V_{\infty}$. We consider the following seven subcases for the construction in $[0,3 / 8] \times[0,1 / 8]$.

( $\left.\mathrm{g}_{\mathrm{b} 1}\right) V_{0} \cap C$ is nonempty and finite. Then we let $A_{2}$ be an admissible staircase in $[0,3 / 8] \times[0,1 / 8]$ with end points $(0,0)$ and $(3 / 8,1 / 8)$.

$\left(\mathrm{g}_{\mathrm{b} 2}\right) V_{0} \cap C=\emptyset$ and $V_{0} \cup\left\{c_{3}\right\}$ is a maximal free arc in $Z$. Then let $A_{2}=(0,0)(3 / 8,1 / 8)$.

(g $\left.\mathrm{g}_{\mathrm{b} 3}\right) V_{0} \cap C=\emptyset$ and $V_{0} \cup\left\{c_{3}\right\}$ is a true cyclic element of $Z$. Then let $A_{2}=[0,1 / 4] \times\{0\}$.

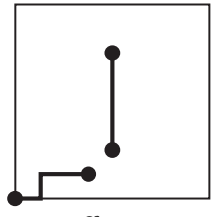

$\mathrm{g}_{\mathrm{b} 1}$

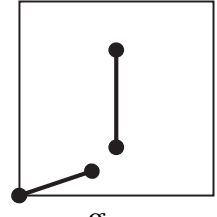

$\mathrm{g}_{\mathrm{b} 2}$

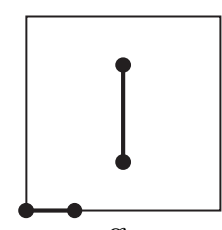

gb3

$\left(\mathrm{g}_{\mathrm{b} 4}\right) V_{\infty} \cap U_{0} \cap C$ is nonempty and finite. Then let $A_{2}$ be an admissible staircase in $[1 / 8,1 / 2] \times[1 / 8,1 / 4]$ with end points $(1 / 8,1 / 8)$ and $(1 / 2,1 / 4)$.

$\left(\mathrm{g}_{\mathrm{b} 5}\right) V_{\infty} \cap U_{0} \cap C=\emptyset$ and $c_{3}$ and $c_{1}$ are end points of a maximal free arc in $Z$. Then let $A_{2}=(1 / 8,1 / 8)(1 / 2,1 / 4)$.

$\left(\mathrm{g}_{\mathrm{b} 6}\right) V_{\infty} \cap U_{0} \cap C=\emptyset$ and $c_{3}$ and $c_{1}$ are points of the same true cyclic element of $X$. Then let $A_{2}=[1 / 4,1 / 2] \times\{1 / 4\}$.
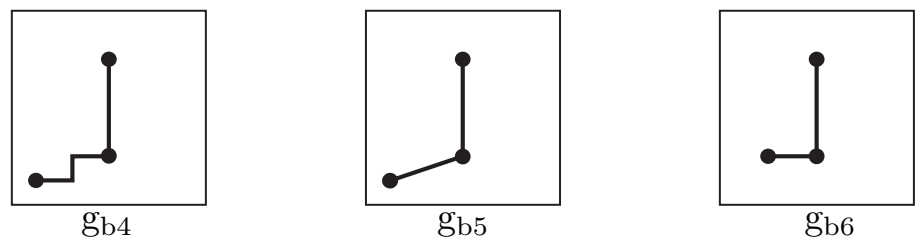

(g $\left.\mathrm{g}_{\mathrm{b} 7}\right) V_{0} \cap C$ and $V_{\infty} \cap U_{0} \cap C$ are both infinite. Then pick $c_{4} \in C$ such that either $\left(\mathrm{g}_{\mathrm{b} 7}^{\prime}\right) c_{3}$ and $c_{4}$ are end points of a maximal free arc, or $\left(\mathrm{g}_{\mathrm{b} 7}^{\prime \prime}\right) c_{3}$ 
and $c_{4}$ are points of the same true cyclic element of $Z$. By the definition of $C$ we can pick $c_{4}$ satisfying either $\left(\mathrm{g}_{\mathrm{b} 7}^{\prime}\right)$ or $\left(\mathrm{g}_{\mathrm{b} 7}^{\prime \prime}\right)$.

If $\left(\mathrm{g}_{\mathrm{b} 7}^{\prime}\right)$ holds, then let $A_{2}=(1 / 16,1 / 16)(7 / 16,3 / 16)$. If $\left(\mathrm{g}_{\mathrm{b} 7}^{\prime \prime}\right)$ holds, then let $A_{2}=[1 / 8,3 / 8] \times\{1 / 8\}$.
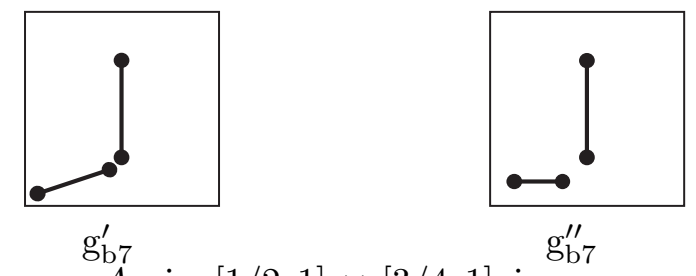

Construct an arc $A_{3}$ in $[1 / 2,1] \times[3 / 4,1]$ in a manner similar to the construction of $A_{2}$ in cases $\left(\mathrm{g}_{\mathrm{b} 1}\right)$ through $\left(\mathrm{g}_{\mathrm{b} 7}\right)$.

In each of the cases above there are one, two, three, or four squares determined by the arcs and the points $(0,0)$ and $(1,1)$. The procedure done in (a) through (g) can be repeated in each of these squares thereby leaving a finite number of squares of at most half the diameter. Thus, an inductive argument results in a set whose closure is the required $\operatorname{arc} B$. The fact that $B$ is an arc can be shown by first showing that it is connected and then showing that for any point in it other than $(0,0)$ and $(1,1)$, the horizontal line in the plane through that point or the vertical line in the plane through that point intersects $B$ only in that point - thus $(0,0)$ and $(1,1)$ are the only two noncut points of $B$ (see Th. 1 of [K, p. 179]).

\section{Examples}

EXAMPLE 1. We describe a diameter level for a metric on a 2-cell which in not locally connected. The example is similar to one in $[\mathrm{P}]$. Let $i=0,1, \ldots$ For $t \in\left[3 / 2^{i+2}, 1 / 2^{i}\right]$, let $S_{t}=\left\{(x, y, t): x^{2}+y^{2}=\left(1+1 / 2^{i}-t\right)^{2}\right\}$. For $t \in\left[1 / 2^{i+1}, 3 / 2^{i+2}\right)$, let $S_{t}=\left\{(x, y, t): x^{2}+y^{2}=\left(1-1 / 2^{i+1}+t\right)^{2}\right\}$. Let $Y=\left\{(x, y, 1): x^{2}+y^{2}<1\right\}$ and $S_{0}=\left\{(x, y, 0): x^{2}+y^{2}=1\right\}$. Let

$$
X=\left[\bigcup_{t \in[0,1]} S_{t}\right] \cup Y .
$$

We will show that any arc in $L_{2}$ from $S_{1 / 2^{i}}$ to $S_{1 / 2^{i+1}}$, where $i \geq 1$, must be of diameter larger than or equal to $\sqrt{2}$.

Let $f$ be a homeomorphism from $[0,1]$ to a subset of $L_{2}$ such that $f(0)=S_{1 / 2^{i}}$ and $f(1)=S_{1 / 2^{i+1}}$. Using cylindrical coordinates, we define a projection $\pi: \bigcup_{t \in[0,1]} S_{t} \rightarrow S_{1 / 2^{i+1}}$ by $\pi(\theta, r, h)=\left(\theta, 1,1 / 2^{i+1}\right)$ where $0 \leq \theta \leq 2 \pi, 1 \leq r \leq 1 \frac{1}{4}$, and $0 \leq h \leq 1$.

We say that two lines $\pi^{-1}(p)$ and $\pi^{-1}\left(p^{\prime}\right)$ are antipodal lines if $p$ and $p^{\prime}$ are antipodal points on $S_{i+1}$. We note the following observation which will be used in our exposition. 
(*) If $\pi^{-1}(p)$ and $\pi^{-1}\left(p^{\prime}\right)$ are antipodal lines and if $q \in \pi^{-1}(p)$ such that $q \notin\left(\bigcup_{i=0}^{\infty} S_{1 / 2^{i}}\right) \cup S_{0}$, then $d\left(q, \pi^{-1}\left(p^{\prime}\right)\right)>2$.

Let $t^{\prime}=\min \left\{t \in[0,1]: f(t)\right.$ contains antipodal points on $\left.S_{1 / 2^{i+1}}\right\}$. There are two cases to consider.

Case 1: $t^{\prime}=1$. Let $0<\varepsilon<1 / 2^{i+2}$. Let $\delta>0$ such that if $t \in(1-\delta, 1]$ then $H_{d}(f(1), f(t))<\varepsilon$. Let $t_{0} \in(1-\delta, 1)$. So, $f\left(t_{0}\right) \subset \bigcup_{p \in S_{1 / 2^{i+1}}} B(p, \varepsilon)$. Clearly, $H_{d}\left(S_{1 / 2^{i+1}}, f\left(t_{0}\right)\right) \geq H_{d}\left(S_{1 / 2^{i+1}}, \pi f\left(t_{0}\right)\right)$. The set $\pi f\left(t_{0}\right)$ must be a connected subset of $S_{1 / 2^{i+1}}$ which properly contains a semicircle, since $f\left(t_{0}\right)$ is connected, $\pi$ is continuous, and $H_{d}\left(S_{1 / 2^{i+1}}, \pi f\left(t_{0}\right)\right)<\sqrt{2}$. Let $p$ and $p^{\prime}$ be antipodal points of $\pi\left(f\left(t_{0}\right)\right)$. Then $\left\{p, p^{\prime}\right\} \nsubseteq f\left(t_{0}\right)$ since $t_{0}<1$. Let $q \in \pi^{-1}(p) \cap f\left(t_{0}\right)$ and $q^{\prime} \in \pi^{-1}\left(p^{\prime}\right) \cap f\left(t_{0}\right)$. By $(*), d\left(q, q^{\prime}\right)>2$. Hence, $\operatorname{diam} f\left(t_{0}\right)>2$. Consequently, $t^{\prime} \neq 1$.

Case 2: $t^{\prime}<1$. Let $q$ and $q^{\prime}$ be antipodal points of $f\left(t^{\prime}\right) \cap S_{1 / 2^{i+1}}$. Let $p \in S_{1 / 2^{i+1}}$ such that $\pi^{-1}(p) \cap f\left(t^{\prime}\right)=\emptyset$. To see that such a point $p$ exists, we make the following observations. If $f\left(t^{\prime}\right) \subset S_{1 / 2^{i+1}}$ then it is clear there exists such a $p$ since $t^{\prime} \neq 1$ and $f\left(t^{\prime}\right) \neq S_{1 / 2^{i+1}}$. If $f\left(t^{\prime}\right) \cap\left(X-S_{1 / 2^{i+1}}\right) \neq \emptyset$ then let $p^{\prime} \in f\left(t^{\prime}\right) \cap\left(\bigcup_{t \in J} S_{t}\right)$ where $J=\left(1 / 2^{i+2}, 1 / 2^{i+1}\right) \cup\left(1 / 2^{i+1}, 1 / 2^{i}\right)$. Clearly, such a point $p^{\prime}$ exists since if $f\left(t^{\prime}\right)$ is connected, $f\left(t^{\prime}\right) \nsubseteq S_{1 / 2^{i+1}}$, and $f\left(t^{\prime}\right) \cap S_{1 / 2^{i+1}} \neq \emptyset$ then $f\left(t^{\prime}\right) \not Y Y \cup\left(\bigcup_{t \in I} S_{t}\right) \cup S_{1 / 2^{i+1}}$, where $I=$ $\left[0,1 / 2^{i+1}\right] \cup\left[1 / 2^{i}, 1\right]$. Let $p$ be the point on $S_{1 / 2^{i+1}}$ which is antipodal to $\pi\left(p^{\prime}\right)$. By $(*), d\left(p, \pi^{-1}\left(\pi\left(p^{\prime}\right)\right)>2\right.$, so $\pi^{-1}(p) \cap f\left(t^{\prime}\right)=\emptyset$. Let $0<\varepsilon<$ $\min \left\{1 / 2^{i+2}, d\left(f\left(t^{\prime}\right), \pi^{-1}(p)\right) / 2\right\}$. Pick $\delta>0$ such that if $t \in\left(t^{\prime}-\delta, t^{\prime}\right]$ then $H_{d}\left(f(t), f\left(t^{\prime}\right)\right)<\varepsilon$. Let $t_{0} \in\left(t^{\prime}-\delta, t^{\prime}\right)$. Note that $\pi^{-1}(p) \cap f\left(t_{0}\right)=\emptyset$, since $d\left(\pi^{-1}(p), f\left(t^{\prime}\right)\right)>2 \varepsilon$ and $\left.H_{d}\left(f\left(t^{\prime}\right)\right), f\left(t_{0}\right)\right)<\varepsilon$.

Let $a \in f\left(t_{0}\right) \cap B(q, \varepsilon)$ and $b \in f\left(t_{0}\right) \cap B\left(q^{\prime}, \varepsilon\right)$. Note that $\pi(a) \in B(q, \varepsilon)$ and $\pi(b) \in B\left(q^{\prime}, \varepsilon\right)$. Let $a^{\prime}$ be the point which is antipodal to $\pi(a)$. If $\pi(a) \neq a$ then $\pi^{-1}\left(a^{\prime}\right) \cap f\left(t_{0}\right)=\emptyset$ by $(*)$ since $a \notin\left(\bigcup_{i=0}^{\infty} S_{1 / 2^{i}}\right) \cup S_{0}$. If $\pi(a)=a$ then $a^{\prime} \notin f\left(t_{0}\right)$ since $t_{0}<t^{\prime}$. Hence, $f\left(t_{0}\right) \cap \pi^{-1}\left(a^{\prime}\right)=\emptyset$ by $(*)$. In either case $f\left(t_{0}\right) \cap \pi^{-1}\left(a^{\prime}\right)=\emptyset$. Let $b^{\prime}$ be the point which is antipodal to $\pi(b)$. Similarly, we see that $f\left(t_{0}\right) \cap \pi^{-1}\left(b^{\prime}\right)=\emptyset$.

Since $f\left(t_{0}\right)$ is connected and $\pi\left(f\left(t_{0}\right)\right) \subset S_{0}-\left\{p, b^{\prime}, a^{\prime}\right\}$ we have either $\pi\left(f\left(t_{0}\right)\right) \varsubsetneqq \widehat{a^{\prime} p}$ where $\widetilde{a^{\prime} p}$ is the arc on $S_{0}$ which does not contain $b^{\prime}$, or $\pi\left(f\left(t_{0}\right)\right) \varsubsetneqq{\widehat{p b^{\prime}}}^{\prime}$ where ${\widehat{p b^{\prime}}}^{\prime}$ is the arc on $S_{0}$ which does not contain $a^{\prime}$, or $\pi\left(f\left(t_{0}\right)\right) \subseteq \widetilde{b^{\prime} a}$ where $\widetilde{b^{\prime}} a$ is the arc on $S_{0}$ which does not contain $p$. Let $\widehat{q q^{\prime}}$ be the arc on $S_{0}$ which does not contain $p$. So, $\bar{b}^{\prime} a^{\prime} \subset \widehat{q q^{\prime}} \cup B(q, \varepsilon) \cup B\left(q^{\prime}, \varepsilon\right)$ and $H_{d}\left(b^{\prime} a^{\prime}, S_{0}\right)>\sqrt{2}-\varepsilon$. Consequently, if $\pi\left(f\left(t_{0}\right)\right) \subset \widetilde{b^{\prime} a^{\prime}}$ then $H_{d}\left(S_{0}, f\left(t_{0}\right)\right) \geq$

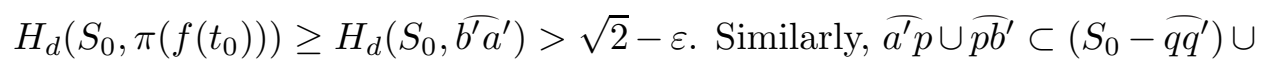
$B(q, \varepsilon) \cup B\left(q^{\prime}, \varepsilon\right)$ and $H_{d}\left(\widehat{a^{\prime} p} \cup \widehat{p b}^{\prime}, S_{0}\right)>\sqrt{2}-\varepsilon$. So, if $\pi\left(f\left(t_{0}\right)\right) \subset \widetilde{a^{\prime} p} \cup \widehat{p b^{\prime}}$ 
then $H_{d}\left(S_{0}, f\left(t_{0}\right)\right) \geq H_{d}\left(S_{0}, \pi\left(f\left(t_{0}\right)\right)\right) \geq H_{d}\left(S_{0}, \widehat{a^{\prime} p} \cup \widehat{p b^{\prime}}\right)>\sqrt{2}-\varepsilon$. Hence, for every $\varepsilon>0$, diam $f([0,1])>\sqrt{2}-\varepsilon$. Consequently, diam $f([0,1]) \geq \sqrt{2}$. Hence, we see that $L_{2}$ is not locally connected.

Example 2. We describe a one-dimensional Peano continuum $X$ which has a non-locally connected diameter level. Let

$$
X=\bigcup_{i=0}^{\infty} S_{1 / 2^{i}} \cup\left[\bigcup_{i=1}^{\infty} \bigcup_{j=1}^{2^{i}} \bigcup_{k=1}^{2} L_{i, j, k}\right] \cup S_{0}
$$

where $S_{1 / 2^{i}}$ is a circle as defined in Example 1 for $i=0,1, \ldots$ and $S_{0}$ is as defined in Example 1. For each pair $(i, j), L_{i, j, 1}$ is the straight line segment joining the points $x_{i, j}$ and $y_{i, j}$ and $L_{i, j, 2}$ is the straight line segment joining the points $y_{i, j}$ and $z_{i, j}$ where

$$
\begin{aligned}
& x_{i, j}=\left((j-1) \pi 2^{1-i}, 1,2^{1-i}\right), \\
& y_{i, j}=\left((j-1) \pi 2^{1-i}, 1+2^{-i-1}, 3 \cdot 2^{-i-1}\right) . \\
& z_{i, j}=\left((j-1) \pi 2^{1-i}, 1,2^{-i}\right) .
\end{aligned}
$$

By a proof similar to the one in the first example, we see that $L_{2}$ of $C(X)$ is not locally connected.

We remark that when $X$ is a one-dimensional absolute retract then all the levels for size maps are locally connected.

\section{References}

[EN] C. Eberhart and S. B. Nadler, Jr., The dimension of certain hyperspaces, Bull. Acad. Polon. Sci. 19 (1971), 1071-1034.

[K] K. Kuratowski, Topology, Vol. II, Academic Press, New York 1966.

[N1] S. B. Nadler, Jr.. Hyperspaces of Sets, Marcel Dekker, New York 1978.

[N2] - Some problems concerning hyperspaces, in: Topology Conference (V.P.I. and S.U.), R. F. Dickman, Jr. and P. Fletcher (eds.), Lecture Notes in Math. 375, Springer, New York 1974, 190-197.

[P] A. Petrus, Contractibility of Whitney continua in $C(X)$, General Topology Appl. 9 (1978), 275-288.

[W] G. Whyburn, Analytic Topology, Amer. Math. Soc. Colloq. Publ. 28, Amer. Math. Soc., Providence, R.I., 1949.

DEPARTMENT OF MATHEMATICS

WEST VIRGINIA UNIVERSITY

MORGANTOWN, WEST VIRGINIA 26505

U.S.A.
DEPARTMENT OF MATHEMATICS UNIVERSITY OF SOUTHWESTERN LOUISIANA

LAFAYETTE, LOUISIANA 70504 